\title{
Camptothecin-Based Regimens for Treatment of Ewing Sarcoma: Past Studies and Future Directions
}

\author{
Lars Wagner \\ Division of Pediatric Hematology/Oncology, Cincinnati Children's Hospital Medical Center, \\ University of Cincinnati College of Medicine, MLC 7015, 3333 Burnet Avenue, Cincinnati, OH 45229, USA \\ Correspondence should be addressed to Lars Wagner, lars.wagner@cchmc.org
}

Received 1 July 2010; Accepted 17 January 2011

Academic Editor: Irene Andrulis

Copyright @ 2011 Lars Wagner. This is an open access article distributed under the Creative Commons Attribution License, which permits unrestricted use, distribution, and reproduction in any medium, provided the original work is properly cited.

New therapies are needed to improve survival for patients with Ewing sarcoma. Over the past decade, camptothecin agents such as topotecan and irinotecan have demonstrated activity against Ewing sarcoma, especially in combination with alkylating agents. Previous studies have shown camptothecin-based combinations to be tolerable outpatient strategies that are attractive for salvage therapy. This paper highlights important issues related to drug dosing, schedule of administration, pharmacokinetics, toxicity, and activity of commonly used camptothecin-based regimens. Also discussed are strategies for incorporating these regimens into therapy for newly diagnosed patients, including several potential possibilities for combination with targeted agents.

\section{Introduction}

Current treatments using interval compression of multiagent chemotherapy combined with surgery and/or radiation can now cure the majority of patients with localized Ewing sarcoma [1]. However, outcomes are much worse for patients who develop disease recurrence after initial therapy, or who have metastases at diagnosis. For example, fewer than $20 \%$ of patients with relapsed Ewing sarcoma are likely to be longterm survivors [2], and patients who present with metastatic bone and/or bone marrow disease at initial diagnosis share a similarly poor prognosis [3]. New therapeutic approaches are clearly necessary to improve overall survival for these patients.

Chemotherapy for Ewing sarcoma has historically consisted of alkylating agents and anthracyclines. Two modifications of this backbone have led to significant improvements in outcome and have helped to define the current standard of care. First, the addition of cassettes of ifosfamide and etoposide onto the 3-drug combination of vincristine, doxorubicin, and cyclophosphamide has improved survival for patients with localized disease [4]. Building on these results, Womer et al. have shown that compression of the interval between treatment courses from 3 weeks to 2 weeks provides additional improvement in 3-year event-free survival, which is now up to $76 \%$ for nonmetastatic patients [1]. Since further modification of this 5-drug regimen seems unlikely to produce additional benefits [5], future regimens will likely need to incorporate new drugs in order to continue progress in the field.

Camptothecin agents have been evaluated in Ewing sarcoma patients for the last decade. This class of agents is attractive because of commercial availability, modest singleagent activity, and demonstrated tolerability and synergy with alkylating agents. Camptothecins exert cytotoxicity by stabilizing the covalent complex between DNA and topoisomerase I, the enzyme which relieves torsional strain of DNA. This stabilization process prevents religation of DNA, and the ensuing collision of the stabilized complex with the advancing replication fork results in double strand breaks and cell death. Preclinical studies have suggested that activity of camptothecins is greatest when given in combination with alkylating agents [6]. Mechanistically, DNA damage from alkylators may increase the importance of topoisomerase I for cell repair, thus rendering tumor cells more sensitive to topoisomerase I inhibition [7]. This activity is also enhanced in preclinical experiments when protracted, low-dose administration is used compared to 
single large doses [8], which is consistent with the S-phasespecific mechanism of action.

This paper summarizes past Ewing sarcoma clinical trials of the two most commonly used camptothecins, topotecan, and irinotecan. Emphasis is placed on differences in their toxicity profiles, schedules, and routes of administration, and partnering alkylating agents. Finally, an outline is provided regarding potential future directions in which these agents may be further developed.

\section{Topotecan}

2.1. Dosing and Schedule of Administration. Topotecan was the first camptothecin to be tested in Phase II trials against Ewing sarcoma. Various schedules of administration have been evaluated, including continuous infusions lasting from 72 hours [9] to 21 days [10]. Although continuous exposure is intuitively attractive for maximizing activity of S-phasespecific drugs, this approach may actually downregulate free topoisomerase I and lead to drug resistance [11]. Therefore, repeated administration on a protracted schedule may be more beneficial [8]. The most commonly used strategy is to administer 5-day courses of topotecan as a daily 30-minute infusion [12]. Dosages studied have ranged from 0.75$3 \mathrm{mg} / \mathrm{m}^{2} /$ day, depending on whether topotecan is given as a single agent or in combination with other chemotherapeutics. As discussed below, the greatest activity for topotecan against Ewing sarcoma has been in combination with cyclophosphamide, in which both drugs are given daily for five days (i.e., $\mathrm{d} \times 5$ schedule) $[13,14]$. Courses generally are repeated every 21 days, and timely administration is facilitated by the concurrent use of filgrastim.

2.2. Pharmacokinetics. There is significant interpatient variability in topotecan pharmacokinetics, with 10 -fold differences in systemic clearance of topotecan lactone, which is the form of topotecan with greatest antitumor activity [15]. This has led investigators at St. Jude Children's Research Hospital to explore targeting the topotecan dose of individual patients to achieve a prespecified systemic exposure that correlated with activity in mouse tumor models. In one study, administration of a standard topotecan dose resulted in achieving the desired systemic exposure in only 53\% of patients. However, when patients underwent real-time pharmacokinetic testing with the first dose and then had subsequent doses modified based on these results, $72 \%$ of patients finished the course within the targeted drug exposure range [15]. This experience, coupled with data from other clinical trials, allowed for the development of a population pharmacokinetic model which used factors such as body surface area, glomerular filtration rate, and age to help predict up to $76 \%$ of interpatient variability in topotecan clearance [16]. However, to date, neither use of this model nor complicated pharmacokinetic targeting has been employed for treatment of Ewing sarcoma patients, and it remains unclear to what extent the variance in systemic exposure affects overall activity of camptothecin agents, particularly when used in combination with other drugs.
2.3. Route of Administration. Intravenous administration is the most common method of topotecan administration; however, it is both costly and inconvenient, particularly when protracted schedules are used. These factors have led to the study of oral topotecan administration. Daw et al. reported that the oral bioavailability of the intravenous preparation given orally was 0.27 and 0.36 when administered in a gelatin capsule [17]. At the maximum tolerated dose (MTD) of $1.8 \mathrm{mg} / \mathrm{m}^{2}$ given daily for five days for two consecutive weeks (i.e., $\mathrm{d} \times 5 \times 2$ ), systemic exposures were similar to those required to achieve responses in mouse models. Further studies have tested oral topotecan in combination with daily oral cyclophosphamide [18], and with both drugs together with gefitinib in a strategy described in greater detail below [19]. Capsule formulation of topotecan is now approved by the FDA for treatment of relapsed lung cancer, based in part on encouraging results from an adult Phase III trial showing similar activity and tolerability when oral dosing of $2.3 \mathrm{mg} / \mathrm{m}^{2} /$ day $\times 5$ was compared to $1.5 \mathrm{mg} / \mathrm{m}^{2} /$ day $\times 5$ given intravenously [20]. There have been no reported Phase II trials of oral topotecan in Ewing sarcoma patients to date.

2.4. Toxicities. The primary dose-limiting toxicity of topotecan is myelosuppression. In single-agent trials, nonhematologic grade 3-4 toxicities such as nausea or dehydration occur in less than $10 \%$ of patients $[9,10]$. Rare events such as fever and mucositis have also been reported to occur with singleagent topotecan. With more protracted schedules in which drug is administered for 5 days for two consecutive weeks (i.e., $\mathrm{d} \times 5 \times 2$ schedule), diarrhea also may be a prominent complication [17].

When topotecan is given in combination with cyclophosphamide at the recommended Phase II dose (topotecan $0.75 \mathrm{mg} / \mathrm{m}^{2} /$ day $\times 5$; cyclophosphamide $250 \mathrm{mg} / \mathrm{m}^{2} /$ day $\times$ 5), myelosuppression remains the predominant toxicity. In the largest reported Phase II experience, 163 (53\%) of 307 courses given to 83 evaluable pediatric patients with recurrent solid tumors resulted in grade 3-4 neutropenia, despite all patients receiving prophylactic filgrastim [13]. However, no infectious deaths were observed, and Grades 3-4 infections were reported in only $11 \%$ of courses. Thrombocytopenia reaching Grades 3-4 was observed in $44 \%$ of all courses.

2.5. Activity against Ewing Sarcoma. Although a sustained complete response was reported in one of 5 Ewing sarcoma xenograft models treated with single-agent topotecan [21], clinical trials of topotecan by itself for recurrent Ewing sarcoma have been relatively disappointing. For example, the response rates for relapsed/refractory Ewing sarcoma patients treated on three Phase II studies using various doses and schedules were $4 \%, 8 \%$, and $10 \%[9,10,12]$. However, combination with cyclophosphamide markedly improves the response rate in this patient population, with two Phase II studies showing response rates of $33 \%$ and $35 \%[13,14]$. In addition, approximately one-third of patients also had stable disease or minor/mixed responses. The success of 
TABLE 1: Summary of selected studies using topotecan-based therapy for patients with relapsed/refractory Ewing sarcoma.

\begin{tabular}{|c|c|c|c|c|c|}
\hline Author [Ref] & $N$ & $\begin{array}{l}\text { Topotecan dose and } \\
\text { schedule }\end{array}$ & Other drugs & $\begin{array}{c}\text { Response Rate } \\
(\text { complete + partial) }\end{array}$ & Comments \\
\hline $\begin{array}{l}\text { Hawkins et al. } \\
{[10]}\end{array}$ & 20 & $\begin{array}{l}0.3 \mathrm{mg} / \mathrm{m}^{2} / \mathrm{d} \text { for } 21 \text {-day } \\
\text { continuous infusion }\end{array}$ & - & $10 \%$ & \\
\hline $\begin{array}{l}\text { Blaney et al. } \\
\text { [9] }\end{array}$ & 3 & $\begin{array}{l}1-1.3 \mathrm{mg} / \mathrm{m}^{2} / \mathrm{d} \text { for } 72 \text {-hour } \\
\text { continuous infusion }\end{array}$ & - & $4 \%$ & \\
\hline $\begin{array}{l}\text { Bernstein et al. } \\
{[12]}\end{array}$ & 36 & $2 \mathrm{mg} / \mathrm{m}^{2} / \mathrm{d} \times 5$ & - & $8 \%$ & $\begin{array}{l}\text { Given as upfront window for } 6 \\
\text { weeks in newly diagnosed } \\
\text { metastatic patients }\end{array}$ \\
\hline $\begin{array}{l}\text { Bernstein et al. } \\
{[12]}\end{array}$ & 37 & $0.75 \mathrm{mg} / \mathrm{m}^{2} / \mathrm{d} \times 5$ & $\mathrm{CPM} 250 \mathrm{mg} / \mathrm{m}^{2} / \mathrm{d} \times 5$ & $57 \% *$ & $\begin{array}{l}\text { Given as upfront window for } 6 \\
\text { weeks in newly diagnosed } \\
\text { metastatic patients }\end{array}$ \\
\hline $\begin{array}{l}\text { Saylors et al. } \\
{[13]}\end{array}$ & 17 & $0.75 \mathrm{mg} / \mathrm{m}^{2} / \mathrm{d} \times 5$ & $\mathrm{CPM} 250 \mathrm{mg} / \mathrm{m}^{2} / \mathrm{d} \times 5$ & $35 \%$ & $\begin{array}{l}50 \% \text { of patients received at least } \\
6 \text { courses before progression }\end{array}$ \\
\hline $\begin{array}{l}\text { Hunold et al. } \\
{[14]}\end{array}$ & 49 & $0.75 \mathrm{mg} / \mathrm{m}^{2} / \mathrm{d} \times 5$ & $\mathrm{CPM} 250 \mathrm{mg} / \mathrm{m}^{2} / \mathrm{d} \times 5$ & $33 \%$ & $\begin{array}{l}\text { Additional } 26 \% \text { with stable } \\
\text { disease }\end{array}$ \\
\hline
\end{tabular}

*In newly diagnosed patients with metastatic disease; CPM, cyclophosphamide.

this combination has resulted in its frequent use for salvage therapy for patients in first relapse.

A pilot study exploring higher doses of these agents was recently reported by Kushner et al., using cyclophosphamide $4200 \mathrm{mg} / \mathrm{m}^{2}$ by 48 -hour infusion together with topotecan $6 \mathrm{mg} / \mathrm{m}^{2}$ by 72-hour infusion [22]. As expected, myelosuppression was more frequent and severe, although retreatment was possible within 28 days. The limited number of Ewing sarcoma patients studied precludes assessment of whether higher doses improve activity for this tumor type. In addition, because alkylating agents may have significant single agent activity [23], determination of the relative contribution of topotecan for treating Ewing sarcoma is difficult. Details of key clinical trials using topotecan for Ewing sarcoma are summarized in Table 1.

\section{Irinotecan}

3.1. Dosing and Schedule of Administration. As with topotecan, scheduling of irinotecan administration for pediatric solid tumors has tried to capitalize on the S-phase-specific mechanism of action, with more protracted schedules having greater preclinical activity [8]. Many early trials used the $\mathrm{d} \times 5 \times 2$ schedule modeled after a pediatric Phase I trial of intravenous irinotecan reported by Furman et al., which established the MTD of $20 \mathrm{mg} / \mathrm{m}^{2} /$ day [24]. However, when compared directly with a $\mathrm{d} \times 5$ schedule using a dose of $50 \mathrm{mg} / \mathrm{m}^{2} /$ day $\times 5$, there was no difference in response rates or incidence of grade 3-4 toxicities in patients with recurrent rhabdomyosarcoma who were receiving intravenous irinotecan together with vincristine as a 6-week upfront window [25]. This is the only pediatric trial which compared two schedules in a randomized fashion, and the results are similar to comparisons of different schedules in colon cancer patients [26]. Although it is possible that the more protracted $d \times 5 \times 2$ schedule may have greater benefit with diseases other than rhabdomyosarcoma, it is likely that these results will be extrapolated to future Ewing sarcoma trials using irinotecan, given the improved patient convenience and decreased costs of shorter treatment schedules.

3.2. Pharmacokinetics. Similar to topotecan, there is up to one log variability in drug exposures between patients receiving irinotecan [24]. Irinotecan is functionally a prodrug, which is converted by endogenous carboxylesterases to the active topoisomerase I poison SN-38. In adults receiving large single doses of irinotecan every 1-3 weeks, metabolism is significantly affected by polymorphisms in UGT1A1, which controls inactivation of SN-38 through the process of glucuronidation [27]. For the approximately $10 \%$ of patients who are homozygous for the UGT1A $1^{*} 28$ genotype, the risk of toxicity is substantial, resulting in an FDA recommendation for dose reduction in starting doses for these patients [28]. Importantly, this polymorphism has had less impact in pediatric patients receiving protracted irinotecan, as increased toxicity has not been associated with $U G T 1 A 1^{*} 28$ in over 180 patients in 4 clinical trials [29-32]. To date, no genetic factors have been identified to reliably predict toxicity in children receiving protracted irinotecan.

3.3. Route of Administration. As with topotecan, there has been interest in oral administration of irinotecan as a way to improve convenience and reduce cost. Three studies of oral irinotecan have been completed and have demonstrated that administration of the intravenous preparation mixed in cran-grape juice, despite its bitter taste, is indeed feasible over multiple courses in pediatric patients [31-34]. Cost savings have been estimated to be up to fivefold when compared to intravenous administration [34]. Most importantly, mean SN-38 exposures for patients treated at the MTD of $60 \mathrm{mg} / \mathrm{m}^{2} /$ day on the $\mathrm{d} \times 5 \times 2$ schedule are similar to those from patients receiving intravenous irinotecan at the MTD of $20 \mathrm{mg} / \mathrm{m}^{2} /$ day $[24,32,33]$. Analogously, exposures from 
patients receiving oral irinotecan at $90 \mathrm{mg} / \mathrm{m}^{2} /$ day correlate with those receiving intravenous irinotecan at $50 \mathrm{mg} / \mathrm{m}^{2} /$ day [31]. These studies show that for patients for whom oral administration is suitable, this strategy can greatly reduce costs and improve convenience, particularly when partner drugs such as temozolomide are also given orally.

3.4. Toxicities. The dose-limiting toxicity of irinotecan is dependent on the schedule used. For shorter administration schedules, such as the single doses given every 1-3 weeks in most adult studies, myelosuppression is dose limiting [26]. In contrast, late-onset diarrhea and abdominal cramping are dose limiting when irinotecan is given on the protracted $\mathrm{d} \times 5 \times 2$ schedule [24]. For the intermediate $\mathrm{d} \times 5$ schedule, both myelosuppression and diarrhea can be seen, although both toxicities are generally modest and manageable [29]. This favorable toxicity profile has allowed for combination with agents like temozolomide, in which the toxicity is primarily hematologic. In fact, the temozolomide + irinotecan combination can generally be given in three week courses without the routine use of filgrastim support $[35,36]$.

Although severe diarrhea from protracted irinotecan occurs in $\leq 20 \%$ of patients, such morbidity can impact compliance [37], even when the tumor is responding to treatment [38]. Recent efforts to reduce irinotecanassociated diarrhea in pediatric patients have included the use of activated charcoal to reduce intestinal absorption of the active metabolite SN-38 [39], as well as prophylactic cephalosporins $[33,40]$. Antibiotics work by reducing the intestinal colonization of Gram-negative aerobic bacteria, which produce the glucuronidase enzyme that converts inactivated SN-38-glucuronide back to active SN-38, causing direct injury to the colon. Cephalosporins such as cefixime have been helpful in allowing 50\% higher doses of oral irinotecan to be tolerable, resulting in improved systemic exposures [33]. Cephalosporin prophylaxis has been used in all three reported trials of oral irinotecan, and also has been used in patients who have experienced severe gastrointestinal toxicity with previous courses of intravenous irinotecan [41]. However, use of antibiotics is not required for all patients receiving standard doses of intravenous irinotecan, and prolonged cephalosporins may be deleterious if given unnecessarily. When used, cephalosporins are started at least two days prior to chemotherapy and continued for at least three days after chemotherapy. Therefore, patients receiving 5 -day courses of irinotecan can be treated with a 10-day course of antibiotics [31], while patients receiving longer schedules of irinotecan are often continued indefinitely on antibiotics [32].

Although most patients receiving irinotecan and temozolomide do not have significant cumulative toxicity, a recent trial using vincristine, oral irinotecan, and temozolomide (VOIT regimen) showed a subset of patients who experienced fatigue, anorexia, and weight loss that resulted in removal from study [31]. This finding has not been reported in previous studies, but does underscore the point that this regimen may not be well tolerated over multiple courses by all patients.
3.5. Activity against Ewing Sarcoma. Although not formally tested against Ewing sarcoma models, there have been reports of complete sustained responses with single-agent irinotecan in xenograft models of primitive neuroectodermal tumor (PNET), which is clinically similar to Ewing sarcoma [42]. Building on this preclinical data, Bisogno et al. reported a response rate of $38 \%$ in 13 patients with recurrent/refractory extraosseous PNET who were treated with protracted intravenous irinotecan given on a $\mathrm{d} \times 5 \times 2$ schedule [38]. However, no responses were seen in 16 patients with recurrent/refractory PNET/ES in a Children's Oncology Group Phase II trial using intravenous irinotecan $50 \mathrm{mg} / \mathrm{m}^{2} /$ day $\times 5$ [29].

It is likely that the greatest antitumor activity of irinotecan will be in combination with other drugs, such as the methylating agent temozolomide. Houghton and colleagues have demonstrated a schedule-dependent synergy between temozolomide and irinotecan, in which methyl groups placed on DNA by temozolomide cause recruitment of topoisomerase I, thus potentiating the cytotoxic effects of irinotecan given at least one hour later [6, 7, 43]. This two-drug combination has now been studied in three trials, with reported response rates of $29 \%, 64 \%$, and $63 \%$ [35, $36,44]$. Collectively, the cumulative results show partial or complete responses in $32(55 \%)$ of 58 patients with recurrent/refractory Ewing sarcoma who have received 5day courses of temozolomide combined with intravenous irinotecan given on the $d \times 5 \times 2$ schedule. Based on this data, temozolomide + irinotecan, like cyclophosphamide + topotecan, has become a widely used regimen for patients with relapsed or refractory Ewing sarcoma. Interestingly, the mechanisms of resistance to these camptothecin agents appear to be different [45], and responses have been reported with one regimen even when disease progression has been noted with the other [35].

Based on the established role of vincristine in treating Ewing sarcoma, as well as the synergy observed in rhabdomyosarcoma patients who are treated with vincristine + irinotecan [41], more recent trials have added vincristine onto the backbone of temozolomide + irinotecan [31, 46]. Use of vincristine may be particularly attractive for newly diagnosed patients, for whom use of a known active agent like vincristine may help reduce the rate of early disease progression when using an upfront-window design [41]. This addition does not seem to significantly change the toxicity profile, although comparison studies with and without vincristine have not been performed. Details of key clinical trials using irinotecan for relapsed/refractory Ewing sarcoma are provided in Table 2.

\section{Comparison of Topotecan-Based versus Irinotecan-Based Regimens}

Because of the relatively small number of patients with recurrent Ewing sarcoma, randomized trials directly comparing regimens have not been performed. Table 3 summarizes available information for commonly used salvage therapies, highlighting toxicities, drug administration factors, 
TABLE 2: Summary of studies using irinotecan-based therapy for patients with relapsed/refractory Ewing sarcoma.

\begin{tabular}{|c|c|c|c|c|c|c|c|}
\hline $\begin{array}{l}\text { Author } \\
{[\text { Ref] }}\end{array}$ & $N$ & IRN dose and schedule & $\begin{array}{c}\text { Route of IRN } \\
\text { administration }\end{array}$ & TEM dose & $\begin{array}{l}\text { Other } \\
\text { drugs }\end{array}$ & $\begin{array}{c}\text { Response rate } \\
(\text { complete }+ \text { partial })\end{array}$ & Comments \\
\hline $\begin{array}{l}\text { Bisogno } \\
\text { et al. [38] }\end{array}$ & 13 & $20 \mathrm{mg} / \mathrm{m}^{2} / \mathrm{d} \times 5 \times 2$ & IV & - & - & $38 \%$ & $\begin{array}{l}\text { Study limited to soft } \\
\text { tissue PNET patients }\end{array}$ \\
\hline $\begin{array}{l}\text { Cosetti } \\
\text { et al. [37] }\end{array}$ & 3 & $20 \mathrm{mg} / \mathrm{m}^{2} / \mathrm{d} \times 5 \times 2$ & IV & - & - & 0 & \\
\hline $\begin{array}{l}\text { Bomgaars } \\
\text { et al. [29] }\end{array}$ & 16 & $50 \mathrm{mg} / \mathrm{m}^{2} / \mathrm{d} \times 5$ & IV & - & - & 0 & \\
\hline $\begin{array}{l}\text { Casey } \\
\text { et al. [36] }\end{array}$ & 20 & $10 \mathrm{mg} / \mathrm{m}^{2} / \mathrm{d} \times 5 \times 2$ & IV & $100 \mathrm{mg} / \mathrm{m}^{2} / \mathrm{d} \times 5$ & - & $63 \%$ & $\begin{array}{l}\text { Median TTP }=8.3 \\
\text { months }\end{array}$ \\
\hline $\begin{array}{l}\text { Wagner } \\
\text { et al. [35] }\end{array}$ & 14 & $10-20 \mathrm{mg} / \mathrm{m}^{2} / \mathrm{d} \times 5 \times 2$ & IV & $100 \mathrm{mg} / \mathrm{m}^{2} / \mathrm{d} \times 5$ & - & $29 \%$ & $\begin{array}{l}50 \% \text { of patients } \\
\text { received at least } 6 \\
\text { courses before } \\
\text { progression }\end{array}$ \\
\hline $\begin{array}{l}\text { Anderson } \\
\text { et al. [44] }\end{array}$ & 25 & $10 \mathrm{mg} / \mathrm{m}^{2} / \mathrm{d} \times 5 \times 2$ & IV & $100 \mathrm{mg} / \mathrm{m}^{2} / \mathrm{d} \times 5$ & - & $60 \%$ & $\begin{array}{l}\text { Median TTP }=5.5 \\
\text { months }\end{array}$ \\
\hline $\begin{array}{l}\text { Wagner } \\
\text { et al. [31] }\end{array}$ & 5 & 35-90 mg/m²/day & $\mathrm{PO}$ & $\begin{array}{c}100-150 \mathrm{mg} / \mathrm{m}^{2} / \mathrm{d} \\
\times 5\end{array}$ & VCR & $40 \% *$ & $\begin{array}{l}\text { Phase I trial using } \\
\text { different doses and } \\
\text { schedules }\end{array}$ \\
\hline $\begin{array}{l}\text { McNall- } \\
\text { Knapp } \\
\text { et al. [46] }\end{array}$ & 1 & $15 \mathrm{mg} / \mathrm{m}^{2} / \mathrm{d} \times 5 \times 2$ & IV & $100 \mathrm{mg} / \mathrm{m}^{2} / \mathrm{d} \times 5$ & VCR & $100 \%(n=1)$ & \\
\hline
\end{tabular}

TABLE 3: Comparison of commonly used regimens for recurrent Ewing sarcoma.

\begin{tabular}{lcllc}
\hline & $\begin{array}{c}\text { Cyclophosphamide }+ \\
\text { topotecan }\end{array}$ & Temozolomide + irinotecan & High-dose ifosfamide & $\begin{array}{c}\text { Ifosfamide + carboplatin }+ \\
\text { etoposide }\end{array}$ \\
\hline Number of patients & 66 & 58 & 37 & 22 \\
Author (Ref) & Saylors et al. [13] & Casey et al. [36] & Wagner et al. [35] \\
& Hunold et al. [14] & Anderson et al. [44] & Ferrari et al. [23] & Van Winkle et al. [47] \\
Primary toxicity & Myelosuppression & Gastrointestinal & Myelosuppression & Myelosuppression \\
Alopecia & Yes & Uncommon & Yes & Yes \\
Myeloid growth factor & Yes & Rarely & Yes & Yes \\
IV access required & Generally & Optional if administered & Yes & Yes \\
Home administration & Uncommon & Common & No & No \\
Response rates & $33-35 \%$ & $29-63 \%$ & $34 \%$ & $48 \%$ \\
(complete + partial) & & & & \\
\hline
\end{tabular}

and response rates. The prioritization of salvage regimens depends on several factors, including individual patient considerations. Temozolomide + irinotecan is generally less myelosuppressive than cyclophosphamide + topotecan and does not usually require myeloid growth factor support. However, some patients may develop diarrhea, fatigue, or weight loss that becomes problematic. Although both regimens can potentially be given orally, there is a larger published pediatric experience with oral irinotecan (summarized in [34]). Home administration of chemotherapy, whether intravenously or oral, is often attractive for patients, and has been well described with temozolomide + irinotecan [35]. Home administration also helps reduce cost, which can be considerable if multiple courses are administered.
At our institution, patient drug costs alone for an individual with body surface area of $1.5 \mathrm{~m}^{2}$ receiving a standard 5day course of cyclophosphamide + topotecan + pegfilgrastim are $\$ 21,966$ (USD), compared to a standard 5-day course of temozolomide + oral irinotecan costing $\$ 17,599$. The total number of courses given to patients with relapsed disease is variable and depends on both response and tolerability. Extended treatment using cyclophosphamide + topotecan (up to 11 courses) and temozolomide + irinotecan (up to 20 courses) has been described, but the benefits of such protracted therapy are unclear. Relapsed patients may also benefit from local control measures such as radiotherapy [48], which in most situations can be administered concurrently with either regimen. 


\section{Further Development of Camptothecins in Treatment of Ewing Sarcoma}

There are two general ways that camptothecin regimens can be incorporated into frontline therapies. The first is to build on standard 5-drug therapy by inserting cassettes of either cyclophosphamide + topotecan, or temozolomide + irinotecan + vincristine. Based on the success of administering standard 5-drug treatment in two-week intervals [1], the Children's Oncology Group (COG) has performed a feasibility trial examining whether cyclophosphamide + topotecan may also be given in intervals shorter than the typical 3 weeks. Published results from this trial are currently pending; however, the Children's Oncology Group has now opened a randomized Phase III trial in which patients with newly diagnosed localized Ewing sarcoma may receive cassettes of vincristine, cyclophosphamide, and topotecan in 2-week intervals interspersed in the standard 5-drug regimen (http://clinicaltrials.gov/\#NCT01231906).

The second strategy is to use camptothecin regimens as a therapeutic backbone on which to add targeted therapies. Several candidate agents have been evaluated either in clinical trials or preclinical experiments and are discussed below.

\section{Combination Strategies}

6.1. Bevacizumab. Bevacizumab is a monoclonal antibody directed against vascular endothelial growth factor, and targets the newly developing blood vessels necessary for sustaining solid tumors. Although originally thought to have antitumor activity by "choking off" the blood supply to tumors, the primary effect of bevacizumab may actually be related to normalization of the tumor-associated vasculature by pruning off immature or poorly formed blood vessels, thereby improving distribution of chemotherapy [49]. This principle was demonstrated in preclinical experiments by Dickson et al., in which intratumoral topotecan concentrations improved following pretreatment with bevacizumab, which corresponded to better antitumor activity [50]. Given the long half-life of bevacizumab, and the demonstration that intratumoral perfusion peaks 2 days after administration and returns to baseline within 7 days, combination with protracted courses of camptothecins seems reasonable. The COG has recently finished a pilot study in which bevacizumab was added to the backbone of vincristine, cyclophosphamide, and topotecan for patients with relapsed/ refractory Ewing sarcoma [51]. This combination was feasible, and future studies are ongoing, such as an institutional trial at Cincinnati Children's Hospital which combines bevacizumab with the VOIT regimen (vincristine, oral irinotecan, and temozolomide) (http://clinicaltrials .gov/\#NCT00786669).

6.2. IGF-1R Antibodies. Signaling through the insulin-like growth factor type I receptor (IGF-1R) is critical for the growth and maintenance of Ewing sarcoma cells, and its inhibition offers an attractive new target for therapy (reviewed in [52]). IGF-1R also has important effects on apoptosis and response to DNA damage. Wang et al. have shown that the concomitant use of an IGF-1R antibody together with irinotecan produced additive effects and resulted in a $93 \%$ reduction in tumor volume in a mouse model of thyroid carcinoma [53]. Clinical trials of anti-IGF-1R antibodies have already demonstrated single-agent activity against relapsed Ewing sarcoma [54], with one of the largest showing $2(13 \%)$ of 16 patients having objective responses, with another $8(50 \%)$ having stable disease for at least 4 months [55]. The COG is now using the IMC-A12 antibody together with multiagent chemotherapy in a study for metastatic rhabdomyosarcoma (http://clinicaltrials.gov/ \#NCT01055314), and combination strategies for Ewing sarcoma are in early development (http://clinicaltrials.gov/ \#NCT01182883).

6.3. Gefitinib. Gefitinib is a small molecule inhibitor of the epidermal growth factor receptor and is used clinically for treatment of lung cancer. Gefitinib competes with camptothecins at the level of the ABCG2 drug efflux pump, causing inhibition of the pump without directly serving as a substrate [56]. This allows gefitinib to reverse irinotecan resistance in vitro [57], even in cell lines that lack expression of the epidermal growth factor receptor. Since ABCG2 is expressed in normal small intestine, administration of gefitinib also improves by threefold the oral bioavailability of irinotecan in mice [56]. This data has led to recent trials of gefitinib + oral irinotecan for refractory pediatric solid tumors [58], as well as gefitinib + oral topotecan and cyclophosphamide in children with relapsed neuroblastoma [19].

6.4. mTOR Inhibitors. Like gefitinib, the mTOR inhibitor rapamycin inhibits ABCG2 without being a substrate of this pump. Accordingly, rapamycin has effectively reversed in vitro resistance to topotecan [59]. Rapamycin is also synergistic with irinotecan in mouse models of colon cancer, perhaps through inhibition of hypoxia-inducible factor-1 alpha [60]. mTOR inhibitors have had some modest singleagent effects against Ewing sarcoma xenografts [61], and a recently opened COG Phase I trial will examine the combination of temozolomide and irinotecan together with the once-weekly mTOR inhibitor temsirolimus (http://clinicaltrials.gov/\#NCT01141244).

6.5. PARP Inhibitors. Poly (ADP-ribose) polymerase (PARP) is a key mediator of DNA base excision repair and recovery from DNA strand breaks and protect cells from the toxicity of various chemotherapy agents. Inhibitors of PARP can effectively enhance the in vitro and in vivo activity of topotecan, irinotecan, and temozolomide against colorectal and lung cancer cell lines and xenograft models [62]. Interestingly, Tentori et al. demonstrated that PARP inhibition not only increased the activity of temozolomide + irinotecan against tumor xenografts, but also reduced intestinal injury and diarrhea in mouse models, thus offering the possibility of improved activity with less toxicity [63]. 
6.6. New Camptothecins in Development. EZN-2208 is a novel polymer-drug conjugate which links the active metabolite of irinotecan ( $\mathrm{SN}-38$ ) with a multiarm polyethylene glycol. This allows for intravenous administration once every three weeks, with sustained SN-38 exposures that are superior to those seen with protracted irinotecan [64]. In experimental models, activity has been seen even in tumors resistant to irinotecan [65]. Pediatric trials of EZN-2208 are set to begin soon.

\section{Conclusions}

Considerable progress has been made in evaluating camptothecin agents for treatment of Ewing sarcoma, and both topotecan and irinotecan clearly are active when combined with alkylating agents. These drugs have several similarities, including superior activity when given on protracted schedules, wide interpatient variability in pharmacokinetics, and the potential for oral administration. Both cyclophosphamide + topotecan and temozolomide + irinotecan are now widely used for patients with recurrent tumors and offer a good chance of response or disease stabilization. Decisions regarding which regimen to use are often based on toxicity and drug administration issues. The activity and tolerability seen with both regimens assures further exploration in upcoming trials, either as cassettes which are incorporated into standard five-drug regimens or as therapeutic backbones on which to add targeted agents.

\section{References}

[1] R. B. Womer, D. C. West, M. D. Krailo, P. S. Dickman, and B. Pawel, "Randomized comparison of every-two-week v. everythree-week chemotherapy in Ewing sarcoma family tumors (ESFT)," Journal of Clinical Oncology, vol. 26, no. 15, 2008, abstract no. 10504.

[2] A. G. Shankar, S. Ashley, A. W. Craft, and C. R. Pinkerton, "Outcome after relapse in an unselected cohort of children and adolescents with Ewing sarcoma," Medical and Pediatric Oncology, vol. 40, no. 3, pp. 141-147, 2003.

[3] B. H. Kushner and P. A. Meyers, "How effective is dose-intensive/myeloablative therapy against Ewing's sarcoma/primitive neuroectodermal tumor metastatic to bone or bone marrow? The Memorial Sloan-Kettering experience and a literature review," Journal of Clinical Oncology, vol. 19, no. 3, pp. 870-880, 2001.

[4] H. E. Grier, M. D. Krailo, N. J. Tarbell et al., "Addition of ifosfamide and etoposide to standard chemotherapy for Ewing's sarcoma and primitive neuroectodermal tumor of bone," New England Journal of Medicine, vol. 348, no. 8, pp. 694-701, 2003.

[5] L. Granowetter, R. Womer, M. Devidas et al., "Doseintensified compared with standard chemotherapy for nonmetastatic ewing sarcoma family of tumors: a Children's Oncology Group study," Journal of Clinical Oncology, vol. 27, no. 15, pp. 2536-2541, 2009.

[6] P. J. Houghton, C. F. Stewart, P. J. Cheshire et al., "Antitumor activity of temozolomide combined with irinotecan is partly independent of O-methylguanine-DNA methyltransferase and mismatch repair phenotypes in xenograft models," Clinical Cancer Research, vol. 6, no. 10, pp. 4110-4118, 2000.
[7] P. Pourquier, J. L. Waltman, Y. Urasaki et al., "Topoisomerase I-mediated cytotoxicity of $\mathrm{N}$-methyl-N'-nitro-Nnitrosoguanidine: trapping of topoisomerase I by the O6methylguanine," Cancer Research, vol. 61, no. 1, pp. 53-58, 2001.

[8] P. J. Houghton, P. J. Cheshire, J. D. Hallman II et al., "Efficacy of topoisomerase I inhibitors, topotecan and irinotecan, administered at IOW dose levels in protracted schedules to mice bearing xenografts of human tumors," Cancer Chemotherapy and Pharmacology, vol. 36, no. 5, pp. 393-403, 1995.

[9] S. M. Blaney, M. N. Needle, A. Gillespie et al., "Phase II trial of topotecan administered as a 72-hour continuous infusion in children with refractory solid tumors: a collaborative pediatric branch, national cancer institute, and Children's Cancer Group study," Clinical Cancer Research, vol. 4, no. 2, pp. 357-360, 1998.

[10] D. S. Hawkins, S. Bradfield, J. A. Whitlock et al., "Topotecan by 21-day continuous infusion in children with relapsed or refractory solid tumors: a Children's Oncology Group study," Pediatric Blood and Cancer, vol. 47, no. 6, pp. 790-794, 2006.

[11] S. D. Desai, T. K. Li, A. Rodriguez-Bauman, E. H. Rubin, and L. F. Liu, "Ubiquitin/26s proteasome-mediated degradation of topoisomerase I as a resistance mechanism to camptothecin in tumor cells," Cancer Research, vol. 61, no. 15, pp. 5926-5932, 2001.

[12] M. L. Bernstein, M. Devidas, D. Lafreniere et al., "Intensive therapy with growth factor support for patients with Ewing tumor metastatic at diagnosis: Pediatric Oncology Group/Children's Cancer Group Phase II Study 9457-a report from the Children's Oncology Group," Journal of Clinical Oncology, vol. 24, no. 1, pp. 152-159, 2006.

[13] R. L. Saylors III, K. C. Stine, J. Sullivan et al., "Cyclophosphamide plus topotecan in children with recurrent or refractory solid tumors: a pediatric oncology group phase II study," Journal of Clinical Oncology, vol. 19, no. 15, pp. 3463-3469, 2001.

[14] A. Hunold, N. Weddeling, M. Paulussen, A. Ranft, C. Liebscher, and H. Jürgens, "Topotecan and cyclophosphamide in patients with refractory or relapsed Ewing tumors," Pediatric Blood and Cancer, vol. 47, no. 6, pp. 795-800, 2006.

[15] V. M. Santana, W. L. Furman, C. A. Billups et al., "Improved response in high-risk neuroblastoma with protracted topotecan administration using a pharmacokinetically guided dosing approach," Journal of Clinical Oncology, vol. 23, no. 18, pp. 4039-4047, 2005.

[16] P. Schaiquevich, J. C. Panetta, L. C. Iacono et al., "Population pharmacokinetic analysis of topotecan in pediatric cancer patients," Clinical Cancer Research, vol. 13, no. 22, pp. 67036711, 2007.

[17] N. C. Daw, V. M. Santana, L. C. Iacono et al., "Phase I and pharmacokinetic study of topotecan administered orally once daily for 5 days for 2 consecutive weeks to pediatric patients with refractory solid tumors," Journal of Clinical Oncology, vol. 22, no. 5, pp. 829-837, 2004.

[18] D. C. Bowers, V. M. Aquino, P. J. Leavey et al., "Phase I study of oral cyclophosphamide and oral topotecan for children with recurrent or refractory solid tumors," Pediatric Blood and Cancer, vol. 42, no. 1, pp. 93-98, 2004.

[19] A. Donfrancesco, M. A. De Ioris, H. P. McDowell et al., "Gefitinib in combination with oral topotecan and cyclophosphamide in relapsed neuroblastoma: pharmacological rationale and clinical response," Pediatric Blood and Cancer, vol. 54, no. 1, pp. 55-61, 2010. 
[20] J. R. Eckardt, J. Von Pawel, J. L. Pujol et al., "Phase III study of oral compared with intravenous topotecan as second-line therapy in small-cell lung cancer," Journal of Clinical Oncology, vol. 25, no. 15, pp. 2086-2092, 2007.

[21] H. Carol, P. J. Houghton, C. L. Morton et al., "Initial testing of topotecan by the Pediatric Preclinical Testing Program," Pediatric Blood and Cancer, vol. 54, no. 5, pp. 707-715, 2010.

[22] B. H. Kushner, K. Kramer, P. A. Meyers, N. Wollner, and N. K. V. Cheung, "Pilot study of topotecan and highdose cyclophosphamide for resistant pediatric solid tumors," Medical and Pediatric Oncology, vol. 35, no. 5, pp. 468-474, 2000.

[23] S. Ferrari, A. B. Del Prever, E. Palmerini et al., "Response to high-dose ifosfamide in patients with advanced/recurrent ewing sarcoma," Pediatric Blood and Cancer, vol. 52, no. 5, pp. 581-584, 2009.

[24] W. L. Furman, C. F. Stewart, C. A. Poquette et al., "Direct translation of a protracted irinotecan schedule from a xenograft model to a phase I trial in children," Journal of Clinical Oncology, vol. 17, no. 6, pp. 1815-1824, 1999.

[25] L. Mascarenhas, E. R. Lyden, P. P. Breitfeld et al., "Randomized phase II window study of two schedules of irinotecan and vincristine in rhabdomyosarcoma patients at first relapse/disease progression," Journal of Clinical Oncology, vol. 26, p. 542s, 2008.

[26] N. E. Schoemaker, I. E. L. M. Kuppens, V. Moiseyenko et al., "A randomised phase II multicentre trial of irinotecan (CPT11) using four different schedules in patients with metastatic colorectal cancer," British Journal of Cancer, vol. 91, no. 8, pp. 1434-1441, 2004.

[27] Y. Fujiwara and H. Minami, "An overview of the recent progress in irinotecan pharmacogenetics," Pharmacogenomics, vol. 11, no. 3, pp. 391-406, 2010.

[28] Camptosar Package Insert, Pfizer, Inc., New York, NY, USA, 2006.

[29] L. R. Bomgaars, M. Bernstein, M. Krailo et al., "Phase II trial of irinotecan in children with refractory solid tumors: a Children's Oncology Group study," Journal of Clinical Oncology, vol. 25, no. 29, pp. 4622-4627, 2007.

[30] C. F. Stewart, J. C. Panetta, M. A. O’Shaughnessy et al., "UGT1A1 promoter genotype correlates with SN-38 pharmacokinetics, but not severe toxicity in patients receiving lowdose irinotecan," Journal of Clinical Oncology, vol. 25, no. 18, pp. 2594-2600, 2007.

[31] L. M. Wagner, J. P. Perentesis, J. M. Reid et al., "Phase I trial of two schedules of vincristine, oral irinotecan, and temozolomide (VOIT) for children with relapsed or refractory solid tumors: a Children's Oncology Group Phase I consortium study," Pediatric Blood and Cancer, vol. 54, no. 4, pp. 538-545, 2010.

[32] L. M. Wagner, J. G. Villahlanca, C. F. Stewart et al., "Phase I trial of oral irinotecan and temozolomide for children with relapsed high-risk neuroblastoma: a new approach to neuroblastoma therapy consortium study," Journal of Clinical Oncology, vol. 27, no. 8, pp. 1290-1296, 2009.

[33] W. L. Furman, K. R. Crews, C. Billups et al., "Cefixime allows greater dose escalation of oral irinotecan: a phase I study in pediatric patients with refractory solid tumors," Journal of Clinical Oncology, vol. 24, no. 4, pp. 563-570, 2006.

[34] L. M. Wagner, "Oral irinotecan for treatment of pediatric solid tumors: ready for prime time?" Pediatric Blood and Cancer, vol. 54, no. 5, pp. 661-662, 2010.
[35] L. M. Wagner, N. McAllister, R. E. Goldsby et al., “Temozolomide and intravenous irinotecan for treatment of advanced Ewing sarcoma," Pediatric Blood and Cancer, vol. 48, no. 2, pp. 132-139, 2007.

[36] D. A. Casey, L. H. Wexler, M. S. Merchant et al., "Irinotecan and temozolomide for Ewing sarcoma: the memorial sloankettering experience," Pediatric Blood and Cancer, vol. 53, no. 6, pp. 1029-1034, 2009.

[37] M. Cosetti, L. H. Wexler, E. Calleja et al., "Irinotecan for pediatric solid tumors: the Memorial Sloan-Kettering experience," Journal of Pediatric Hematology/Oncology, vol. 24, no. 2, pp. 101-105, 2002.

[38] G. Bisogno, R. Riccardi, A. Ruggiero et al., "Phase II study of a protracted irinotecan schedule in children with refractory or recurrent soft tissue sarcoma," Cancer, vol. 106, no. 3, pp. 703707, 2006.

[39] G. C. Sergio, G. M. Félix, and J. V. Luis, "Activated charcoal to prevent irinotecan-induced diarrhea in children," Pediatric Blood and Cancer, vol. 51, no. 1, pp. 49-52, 2008.

[40] L. M. Wagner, K. R. Crews, C. F. Stewart et al., "Reducing irinotecan-associated diarrhea in children," Pediatric Blood and Cancer, vol. 50, no. 2, pp. 201-207, 2008.

[41] A. S. Pappo, E. Lyden, P. Breitfeld et al., "Two consecutive phase II window trials of irinotecan alone or in combination with vincristine for the treatment of metastatic rhabdomyosarcoma: the Children's Oncology Group," Journal of Clinical Oncology, vol. 25, no. 4, pp. 362-369, 2007.

[42] G. Vassal, M. J. Terrier-Lacombe, M. C. Bissery et al., "Therapeutic activity of CPT-11, a DNA-topoisomerase I inhibitor, against peripheral primitive neuroectodermal tumour and neuroblastoma xenografts," British Journal of Cancer, vol. 74, no. 4, pp. 537-545, 1996.

[43] V. J. Patel, G. B. Elion, P. J. Houghton et al., "Scheduledependent activity of temozolomide plus CPT-11 against a human central nervous system tumor-derived xenograft," Clinical Cancer Research, vol. 6, no. 10, pp. 4154-4157, 2000.

[44] P. Anderson, L. Kopp, N. Anderson et al., "Novel bone cancer drugs: investigational agents and control paradigms for primary bone sarcomas (Ewing's sarcoma and osteosarcoma)," Expert Opinion on Investigational Drugs, vol. 17, no. 11, pp. 1703-1715, 2008.

[45] P. J. Houghton, P. J. Cheshire, J. C. Hallman, M. C. Bissery, A. Mathieu-Boue, and J. A. Houghton, "Therapeutic efficacy of the topoisomerase I inhibitor 7-ethyl-10-(4-[1piperidino]-1-piperidino)-carbonyloxy-camptothecin against human tumor xenografts: lack of cross-resistance in vivo in tumors with acquired resistance to the topoisomerase I inhibitor 9-dimethylaminomethyl-10-hydroxycamptothecin," Cancer Research, vol. 53, no. 12, pp. 2823-2829, 1993.

[46] R. Y. McNall-Knapp, C. N. Williams, E. N. Reeves, R. L. Heideman, and W. H. Meyer, "Extended phase I evaluation of vincristine, Irinotecan, temozolomide, and antibiotic in children with refractory solid tumors," Pediatric Blood and Cancer, vol. 54, no. 7, pp. 909-915, 2010.

[47] P. Van Winkle, A. Angiolillo, M. Krailo et al., "Ifosfamide, Carboplatin, and Etoposide (ICE) reinduction chemotherapy in a large cohort of children and adolescents with recurrent/refractory sarcoma: the Children's Cancer Group (CCG) experience," Pediatric Blood and Cancer, vol. 44, no. 4, pp. 338$347,2005$.

[48] E. A. Czyzewski, S. Goldman, A. J. Mundt, J. Nachman, C. Rubin, and D. E. Hallahan, "Radiation therapy for consolidation of metastatic or recurrent sarcomas in children treated with intensive chemotherapy and stem cell rescue. A feasibility 
study," International Journal of Radiation Oncology, Biology, Physics, vol. 44, no. 3, pp. 569-577, 1999.

[49] R. K. Jain, "Normalization of tumor vasculature: an emerging concept in antiangiogenic therapy," Science, vol. 307, no. 5706, pp. 58-62, 2005.

[50] P. V. Dickson, J. B. Hamner, T. L. Sims et al., "Bevacizumabinduced transient remodeling of the vasculature in neuroblastoma xenografts results in improved delivery and efficacy of systemically administered chemotherapy," Clinical Cancer Research, vol. 13, no. 13, pp. 3942-3950, 2007.

[51] P. Leavey, J. L. Glade-Bender, L. Mascarenhas et al., "Feasibility of bevacizumab combined with vincristine, topotecan, and cyclophosphamide in patients with first recurrent Ewings sarcoma: a Children's Oncology Group study," Journal of Clinical Oncology, vol. 28, no. 15, supplement, 2010, abstract no. 9552.

[52] D. Olmos, D. S. W. Tan, R. L. Jones, and I. R. Judson, "Biological rationale and current clinical experience with antiinsulin-like growth factor 1 receptor monoclonal antibodies in treating sarcoma: twenty years from the bench to the bedside," Cancer Journal, vol. 16, no. 3, pp. 183-194, 2010.

[53] Z. Wang, G. Chakravarty, S. Kim et al., "Growth-inhibitory effects of human anti-insulin-like growth factor-1 receptor antibody (A12) in an orthotopic nude mouse model of anaplastic thyroid carcinoma," Clinical Cancer Research, vol. 12, no. 15, pp. 4755-4765, 2006.

[54] S. Malempati, B. Weigel, A. M. Ingle et al., "A phase I trial and pharmacokinetic study of IMC-A12 in pediatric patients with relapsed/refractory solid tumors: a Children's Oncology Group phase I consortium study," Journal of Clinical Oncology, vol. 27, 2009, abstract no. 10013.

[55] D. Olmos, S. Postel-Vinay, L. R. Molife et al., "Safety, pharmacokinetics, and preliminary activity of the anti-IGF-1R antibody figitumumab (CP-751,871) in patients with sarcoma and Ewing's sarcoma: a phase 1 expansion cohort study," The Lancet Oncology, vol. 11, no. 2, pp. 129-135, 2010.

[56] C. F. Stewart, M. Leggas, J. D. Schuetz et al., "Gefitinib enhances the antitumor activity and oral bioavailability of irinotecan in mice," Cancer Research, vol. 64, no. 20, pp. 74917499, 2004.

[57] S. Nagashima, H. Soda, M. Oka et al., "BCRP/ABCG2 levels account for the resistance to topoisomerase I inhibitors and reversal effects by gefitinib in non-small cell lung cancer," Cancer Chemotherapy and Pharmacology, vol. 58, no. 5, pp. 594-600, 2006.

[58] W. L. Furman, F. Navid, N. C. Daw et al., "Tyrosine kinase inhibitor enhances the bioavailability of oral irinotecan in pediatric patients with refractory solid tumors," Journal of Clinical Oncology, vol. 27, no. 27, pp. 4599-4604, 2009.

[59] A. Gupta, Y. Dai, R. R. Vethanayagam et al., "Cyclosporin A, tacrolimus and sirolimus are potent inhibitors of the human breast cancer resistance protein (ABCG2) and reverse resistance to mitoxantrone and topotecan," Cancer Chemotherapy and Pharmacology, vol. 58, no. 3, pp. 374-383, 2006.

[60] E. Pencreach, E. Guérin, C. Nicolet et al., "Marked activity of irinotecan and rapamycin combination toward colon cancer cells in vivo and in vitro is mediated through cooperative modulation of the mammalian target of rapamycin/hypoxiaInducible factor- $1 \alpha$ axis," Clinical Cancer Research, vol. 15, no. 4, pp. 1297-1307, 2009.

[61] P. J. Houghton, C. L. Morton, E. A. Kolb et al., "Initial testing (stage 1) of the mTOR inhibitor rapamycin by the Pediatric Preclinical Testing Program," Pediatric Blood and Cancer, vol. 50, no. 4, pp. 799-805, 2008.
[62] C. R. Calabrese, R. Almassy, S. Barton et al., "Anticancer chemosensitization and radiosensitization by the novel poly(ADP-ribose) polymerase-1 inhibitor AG14361," Journal of the National Cancer Institute, vol. 96, no. 1, pp. 56-67, 2004.

[63] L. Tentori, C. Leonetti, M. Scarsella et al., "Inhibition of poly(ADP-ribose) polymerase prevents irinotecan-induced intestinal damage and enhances irinotecan/temozolomide efficacy against colon carcinoma," The FASEB Journal, vol. 20, no. 10, pp. 1709-1711, 2006.

[64] Z. Guo, J. J. Wheler, A. Naing et al., "Clinical pharmacokinetics of EZN-2208, a novel anticancer agent, in patients with advanced malignancies: a phase i, first-in-human, doseescalation study," Journal of Clinical Oncology, vol. 26, 2008, abstract no. 2556.

[65] P. Sapra, H. Zhao, M. Mehlig et al., "Novel delivery of SN38 markedly inhibits tumor growth in xenografts, including a camptothecin-11-refractory model," Clinical Cancer Research, vol. 14 , no. 6 , pp. 1888-1896, 2008. 


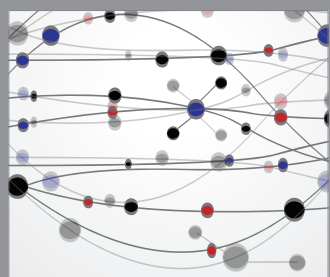

The Scientific World Journal
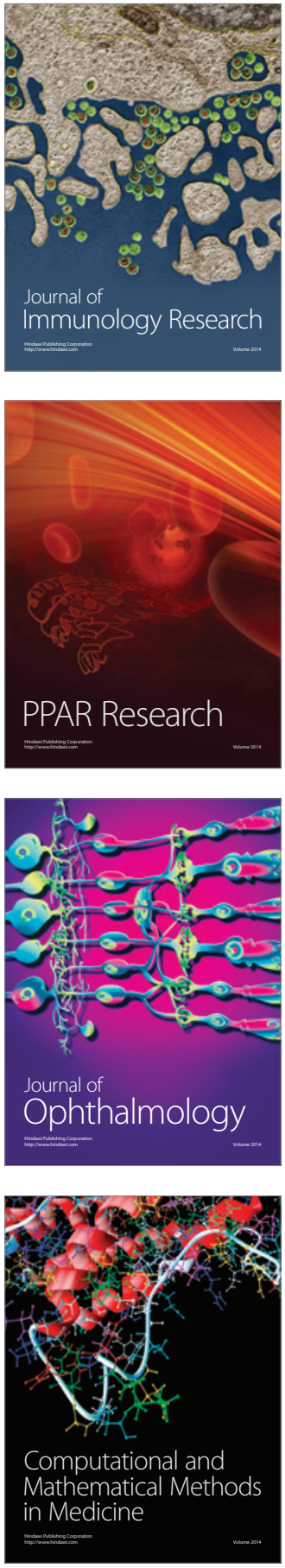

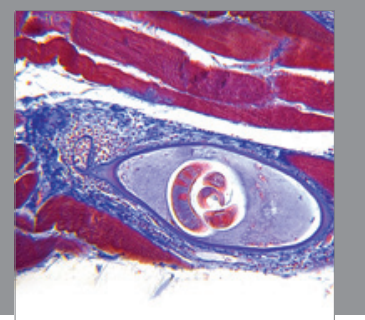

Gastroenterology

Research and Practice
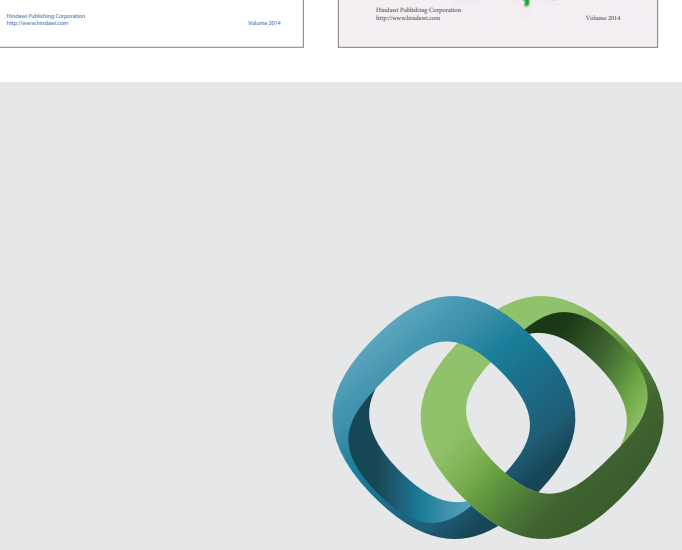

\section{Hindawi}

Submit your manuscripts at

http://www.hindawi.com
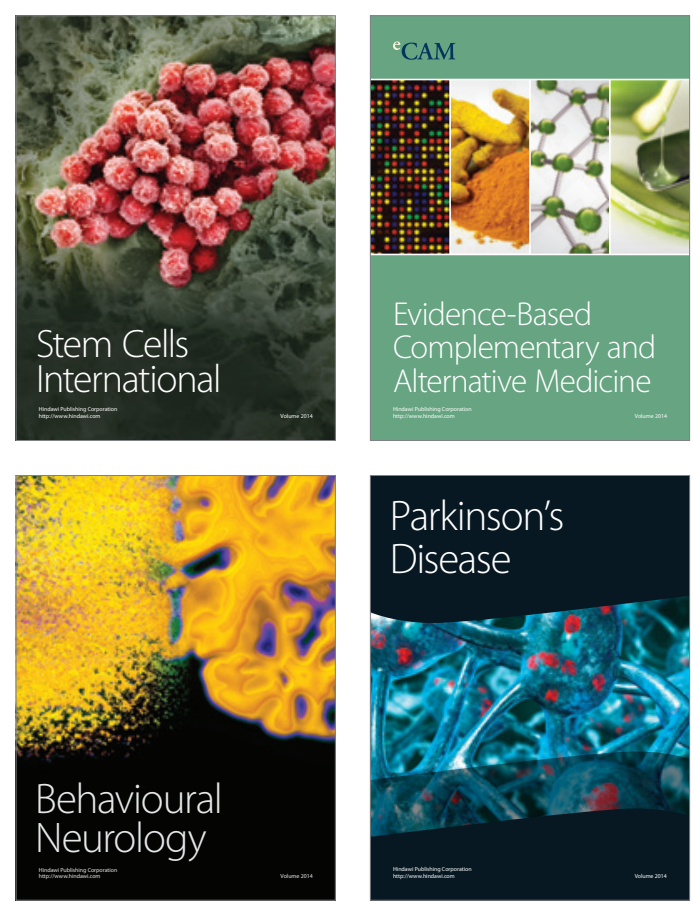

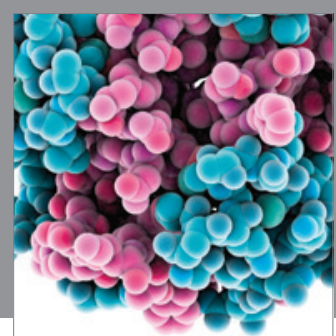

Journal of
Diabetes Research

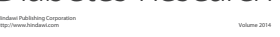

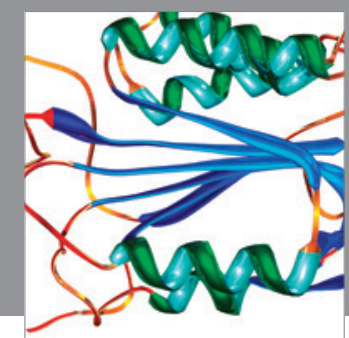

Disease Markers
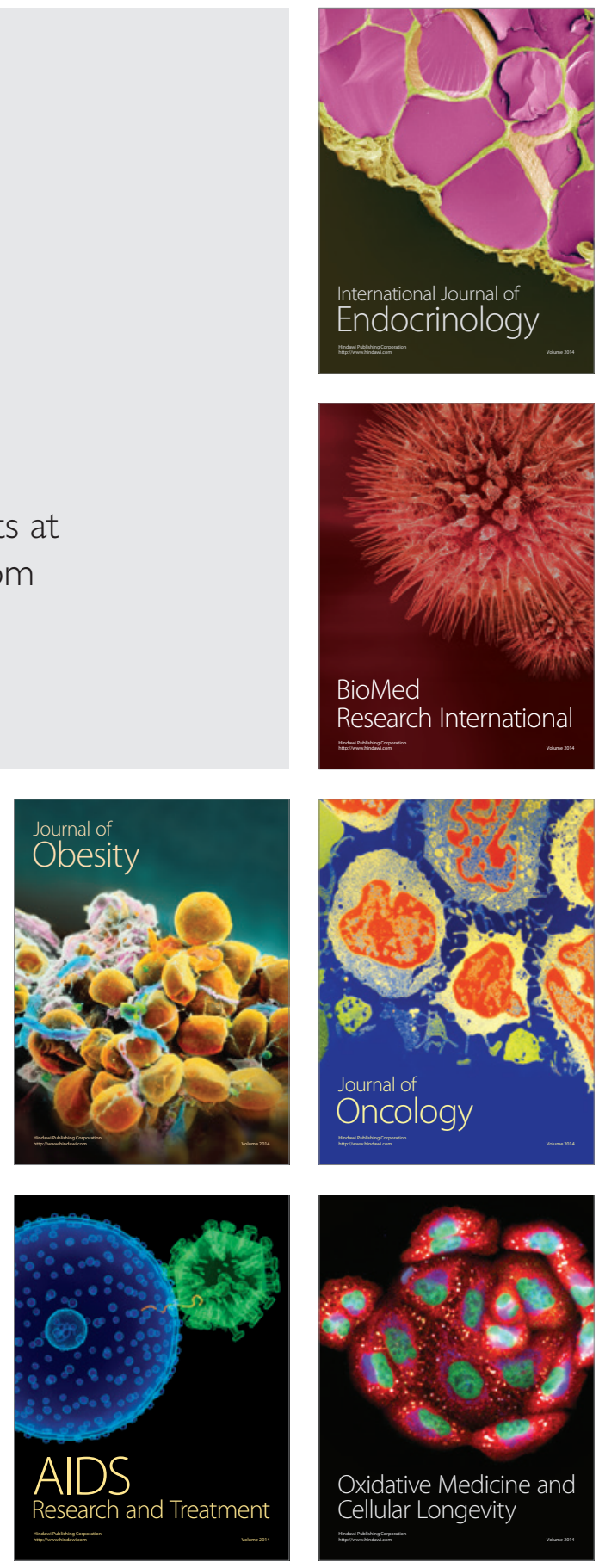